\title{
EL EMPRENDIMIENTO SOCIAL Y EL ESTADO DE BIENESTAR \\ COMO MOTOR DE CAMBIO EN LA SOCIEDAD: EL CASO DE CIUDAD JUÁREZ
}

Flor Rocio Ramirez Martínez/

Universidad Autónoma de

Ciudad Juárez

RECIBIDO: 3 DE JUNIO DE 2016 Aceptado: 8 De Septiembre de 20 i 6

\section{RESUMEN}

D el presente trabajo se

4 aborda la tipología del

$\mathcal{L}$ Estado de bienestar, para encuadrarlo en la complejidad social del municipio fronterizo de Juárez. Se establecen las concepciones teóricas y las características productivas, demográficas, económicas y sociales de dicha zona con el propósito de alinear los esquemas de participación pública con la sociedad, su carácter distributivo y la evolución que ha tenido desde finales del siglo pasado hasta nuestros días, terminando con la con- cepción del emprendimiento social como un camino hacia un nuevo enfoque en el estado de bienestar.

Palabras clave: Emprendimiento social, estado de bienestar, programas sociales.

\section{Abstract \\ 7 his current paper brings up the typol- ogy of the welfare} state in order to classify it in the social complexity of the border town of Ciudad Juarez, Chihuahua. Several theoretical conceptions are established as long as several productive, demographic, and economic 
features of the aforementioned city, with the purpose of matching up the public participation schemes with the society, its distributive character, and the evolution that has taken place since the ending of the past century until these days, finishing with the social entrepreneurship concept as a new path towards a new point of view of the welfare state.

Key words: Social entrepreneurship, welfare state, social programs.

\section{INTRODUCCIÓN}

n Ciudad Juárez la
problemática que ocu-
pan los temas nacionales comunes se amplían aún más por su carácter de ciudad fronteriza. La llegada de miles de personas provenientes de otros lugares del país ha generado un replanteamiento social, demográfico y cultural que ha convertido a esta importante frontera del país en un caleidoscopio tan plural y diverso como demandante.

Según el más reciente Censo del Instituto Nacional de Estadística y Geografía (INEGI, 2010) en Ciudad Juárez viven 1332131 personas, con un tamaño promedio de los hogares de 3.7 habitantes. El ingreso bruto del municipio en 2013 fue de 3800907 (miles de pesos) y la inversión pública ejercida en desarrollo social fue de 1825230 (miles de pesos).

Ciudad Juárez contiene elementos tan nutridos y diferentes que le otorgan una condición especial. La prestación de servicios tiene una demanda acelerada creciente, las políticas públicas sociales se amplían a la gama más diversa imaginable y van desde espacios públicos, centros de desarrollo comunitario, atención de la salud, seguridad, empleo, migración, educación y vivienda.

Adicionalmente, la movilidad social que presenta la convierte en polo de crecimiento plural, que comprende cuestiones de carácter religioso, de identidad y sentido de pertenencia, lo que propicia entre sus nuevos habitantes emociones por lo que dejaron en sus lugares de origen y lo nuevo que han adquirido al pasar a formar parte de su población.

Los miles de nuevos habitantes que arriban cada año son reflejo de las diversas regiones del país y encuentran en Ciudad Juárez asiento, lo que nutre la identidad de los juarenses. Asimismo, mantienen convivencia con la frontera de El Paso, Texas, en donde intervienen cuestiones laborales, culturales e incluso de usos y costumbres. Esta serie de elementos conjuga una pertenencia especial, que ha cimentado su arraigo y le da a la ciudad un rostro propio.

En los últimos cuarenta años, Ciudad Juárez ha recibido una importante cantidad de personas que han encontrado una oportunidad laboral debido a la existencia de la industria maquiladora, aunque este empleo no es necesariamente bien pagado. Estos trabajadores arriban a la ciudad buscando "un futuro mejor", pero al llegar empiezan a solicitar a la ciudad infraestructura y servicios.

A la demanda creciente de nuevos moradores no ha correspondido una asignación presupuestal que solvente sus requerimientos. Los recursos del municipio no aumentan en la proporción de la demanda. Las concentraciones fronterizas, particularmente Ciudad Juárez, no pueden por su complejidad compararse 
con ninguna otra ciudad del país. Es necesario que reciban un tratamiento especial en las asignaciones presupuestales y en los programas sociales para no ocasionar un retraso en el desarrollo social de la ciudad.

Esta panorámica representa un espejo que refleja el concepto del Estado de bienestar aplicable en Ciudad Juárez, para atender y promover iniciativas, recursos y gestiones en el rubro social, considerando una demanda creciente de servicios y una multiplicación de factores socioeconómicos, demográficos y culturales que observan un ritmo más acelerado que la mayoría de las regiones del país y es en donde toma sentido el emprendimiento social.

\section{Marco Teórico}

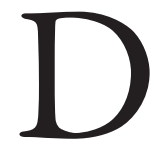
e los tres mil kilómetros de frontera que tiene México con Estados Unidos, Ciudad Juárez representa una zona limítrofe de enorme trascendencia por la mano de obra laboral, el flujo de mercancías, la importancia económica y comercial y el dinamismo de sus ciudades.

El concepto de Estado de bienestar es aplicable en la se- gunda década del siglo XXI, tal como lo fue hace un siglo. Las concepciones teórico-sociales ubican con efectividad esquemas de participación con predomino público que alientan lo privado y lo social en una gama de compromisos que favorecen la distribución y el desarrollo.

Para Martínez (2000), el Estado de bienestar debe estar conformado en primer lugar por una serie de gastos en bienes preferentes como educación, salud, vivienda y empleo; luego por un segundo grupo en programas de transferencias de recursos tales como pensiones, seguros de enfermedad, seguros de desempleo y subsidios familiares, entre otros. Se intentan cubrir riesgos individuales por el conjunto de la sociedad. Estos riesgos son la indigencia, el desempleo, la vejez, la invalidez o la carencia de una educación mínima. Cuando un estado intenta garantizar estos riesgos, estamos ante un Estado del bienestar.

El Estado de bienestar trata de satisfacer las necesidades primarias como la salud, la educación y un empleo que proporcione lo mínimo necesario para vivir. Los antece- dentes de la intervención del Estado en este tema se remontan al canciller Bismark, quien crea las políticas sociales creadas por el Estado.

Después de la Segunda Guerra Mundial, surge la necesidad de que el Estado intervenga no solo en el plano económico, sino en el social. Para Moreno (2001), se puede hablar de los orígenes del Estado de bienestar a partir de la legislación social alemana en el periodo de gobierno del canciller, estadista y militar Otto von Bismarck, quien a fines del siglo XIX aprueba la primera legislación social alemana, que entre sus leyes incluía la ley de seguro de enfermedad y maternidad de 1833 , la ley de accidentes de trabajo de 1884 y la ley de jubilación y defunción de 1889.

Villarrespe (2002) señala que en el siglo XIX la Revolución Industrial introdujo un cambio de percepción sobre la pobreza, asimilando a los pobres con los obreros industriales, es decir, tratando de incluirlos en la vida productiva, ya que se tenía la idea de que pobreza era igual a delincuencia. Debido a esta situación se implementaron controles 
para garantizar la honestidad, la dignidad y el apego social al trabajo de las personas menos favorecidas.

Para el autor lo esencialmente importante es que el problema de los pobres se integra de forma estructural al Estado. La caridad decreció cuando el Estado asumió un nuevo centralismo con las siguientes variantes: a) como represor de vagos, b) como patrón de los verdaderamente necesitados, avalándolos en las posibilidades de empleo y, ocasionalmente, como proveedor de trabajos públicos, y c) como recopilador de información acerca de los pobres.

A finales del siglo XIX, según la revisión de literatura, empiezan a generarse opiniones sobre el Estado de bienestar. Por ejemplo, según documenta Krmpotic (2002), en el Congreso Internacional de Asistencia Pública y Privada, celebrado en París en 1889, se plantea el sostenimiento de la intervención del Estado como contralor de las instituciones privadas, y se empieza a considerar el asistencialismo como una actividad más racional.

Moreno (2001) indica que las bases del Estado de bienestar en Estados Unidos se establecieron después de la gran depresión de 1929 y sobre todo con la aprobación, en 1935, de la "Social Security Act", aunque posteriormente unos programas sociales menos comprensivos y la carencia de un sistema nacional de salud pública, han configurado al "welfare" estadounidense como un referente de mínimos dentro de lo que se conoce como modelo anglosajón.

El sociólogo Esping-Andersen, apoyado en los planteamientos de Marshall, se basa en el concepto de ciudadanía social para desarrollar su propio concepto sobre el Estado de bienestar. Esping-Andersen (1993) construyó una tipología que identifica tres tipos de Estados de bienestar, según su peso e intensidad redistributiva, como más abajo se indica. De acuerdo con el autor, un régimen de bienestar es una combinación institucional de producción mixta de bienestar entre el Estado, la familia, el mercado y la sociedad civil, que depende de tres factores: la naturaleza de la movilización de las clases sociales, las estructuras de coalición de la clase política y el legado histórico. a) El liberal-anglosajón: es característico de los países anglosajones. No focaliza los programas. Estados Unidos es un ejemplo donde el mercado es fundamental. El estado interviene poco y no es su objetivo redistribuir la riqueza. Los programas sociales solo cubren a la población más pobre, los subsidios son modestos y duran poco, la salud es mayoritariamente privada, con programas públicos solo para jubilados o con ingresos muy bajos. El mercado de trabajo está desregulado, con salarios mínimos irrisorios y escasa protección laboral.

b) El socialdemócrata: desarrollado en los países escandinavos, se fundamenta en la búsqueda de la protección pública integral con una tendencia al igualitarismo. En ese sentido, considera un alta provisión de servicios sociales y se centra en el Estado. Los programas sociales son extensivos, están bien financiados y cubren a toda la población. El resultado de este modelo es el de sociedades extremadamente igualitarias, con 
altos niveles de movilidad social, muy bajos niveles de pobreza y envidiables niveles de competitividad e innovación industrial, gracias a una mano de obra bien calificada.

c) El conservador: En este modelo se incluyen países como Alemania, Francia y Bélgica. La intervención del Estado es considerable, sin embargo, la disposición redistributiva es limitada. Como los programas universales no acostumbran tener una buena calidad, los ciudadanos de clase alta recurren al sector privado. En este modelo existen agresivas políticas sociales de empleo y guarderías gratuitas, con mercados de trabajo muy regulado a fin de proteger a los que tienen empleo.

Para Pico (1999), el Estado de bienestar comienza con mayor auge a partir de 1945 , cuando la mayoría de los países capitalistas adoptan la doctrina del Reporte Beveridge y los postulados de política macroeconómica de Keynes. La teoría keynesiana intentaba disminuir los efectos de la depresión actuando sobre la demanda agregada a través del Estado. De ahí que la expansión de los programas de bienestar actuados desde arriba se justificaban no solo con el fin de cubrir las necesidades básicas de la población, sino también como política económica para regular el mercado y reavivar el consumo. El Reporte Beveridge fue creado por Willian Beveridge, de nacionalidad inglesa, en 1942 . Beveridge deseaba que después de la guerra se eliminara la desigualdad social, por lo que el Estado debería garantizar la seguridad social.

Según Bustelo \& Minujin (1997), el Reporte Beveridge planteaba la convicción de evitar que después de la guerra se retomara el sistema de desigualdades sociales del pasado, promoviendo un programa completo de seguros sociales para trabajadores y no trabajadores que cubriera la desocupación, la incapacidad, la maternidad, la viudez, etcétera. Asimismo, proponía la intervención estatal en la seguridad social y el otorgamiento de subsidios a las clases más necesitadas. A partir del reporte, se abandona la idea de
Bismark del seguro social solo para trabajadores, para pasar a la idea de una protección mínima para todos, independientemente de si las personas contribuyeron o no.

Sen (2002) señala que en ocasiones las enfermedades, la pérdida del trabajo, la discapacidad o el bajo nivel salarial pueden ser motivo para que las personas se hundan en la pobreza extrema, y es entonces cuando el Estado de bienestar apoya para solucionar en parte sus necesidades. Según el autor, en la Europa de los años veinte a los cuarenta o principios del periodo de posguerra, la expectativa de vida era muy baja, aunado a la incidencia de enfermedades y pobreza. Ahora, gracias al Estado de bienestar generado por la asistencia sanitaria, la seguridad social, los programas contra la pobreza, el seguro del desempleo y los planes de pensión, la expectativa de vida ha cambiado radicalmente. Para Sen, el Estado de bienestar ha sido quizás la mayor aportación de la civilización europea al mundo.

Durante los años setenta se gestó lo que puede llamarse una "crisis del Estado de bienestar". La escasez de recursos, 
el aumento de la inflación y el cambio del patrón oro-dólar comenzaron la crisis. Los críticos más importantes de la época, Crozier, Huntington, \& Watanuky (1975), se refirieron a la crisis fiscal de los estados de bienestar como una de democracia, ya que señalaban que el gobierno estaba adquiriendo demasiadas cargas sociales, lo que significaba déficit público; esto, aunado a problemas con los sindicatos, los hizo considerar el Estado de bienestar como inviable.

Para Mulas-Granados (2010), la crisis económica internacional que se ha tenido en los países desarrollados ha agudizado la crisis del Estado de bienestar, originalmente generada por la crisis económica de la década de los años setenta. De tal forma que se hace necesario plantear diferentes alternativas para afrontar esta crisis, así como las nuevas demandas y riesgos sociales que comienzan a aparecer en las actuales sociedades desarrolladas, tales como inmigración, acceso a la vivienda, envejecimiento y desempleo, entre otros.

El modelo liberal o anglosajón que se expuso anterior- mente se retoma en la década de los 90 a través de la contracción "workfare" o "welfare to work", sobre todo en Estados Unidos y Gran Bretaña. Según Lodemel y Trickey (2001), el "workfare" estaría compuesto por programas o medidas que requieren el retorno al mercado laboral para que la persona pueda cobrar las prestaciones sociales. En Estados Unidos esta reforma la realiza el Presidente Bill Clinton en 1996 y en Inglaterra el primer ministro Anthony Blair, quienes eliminan apoyos y otorgan prioridad al empleo, condicionando las ayudas que pudiera otorgar el Estado a tener un trabajo.

Las llamadas "rentas mínimas", mejor conocidas en México como "subsidios", surgieron con la crisis del Estado del bienestar, cuando las personas perdían sus empleos. $\mathrm{O}$ se dejaba a las personas fuera de la asistencia social o se debía reformar el modelo de protección social. Es ahí donde se crean estos subsidios para grupos vulnerables, los cuales hoy en día en muchos de los países, sobre todo latinoamericanos, se han convertido en una nue- va manera de reestructurar el Estado de bienestar.

Para Euzéby (1991), los subsidios son complementarios a la seguridad social, son la última opción de seguridad para ciudadanos de escasos recursos y son de carácter condicional. De este modo, hay que cumplir ciertas exigencias como por ejemplo la edad, la residencia o la nacionalidad, con el compromiso a participar en programas de inserción.

Moreno (2008) señala que inicialmente los subsidios son creados para las personas que no han cotizado al sistema de Seguridad Social, que lo han hecho de forma insuficiente o que no cumplen algunos de los requisitos establecidos. Es decir, que nacen con el objetivo de cubrir los "agujeros" que deja el sistema contributivo dentro del Estado del bienestar. A pesar de esto, con el paso del tiempo los subsidios han sido una pieza fundamental en la lucha contra la vulnerabilidad y la pobreza.

\section{Ciudad Juárez: una EXPERIENCIA PARA EL ESTADO DE BIENESTAR n Ciudad Juárez los $\checkmark$ subsidios han sido fun-}


cir la pobreza. Esta frontera, cuyo principal motor es la industria maquiladora que se nutre de mano de obra productiva y especializada de todas partes del país (IMIP, 2003), en los últimos 40 años ha cuadriplicado su población con tasas de crecimiento demográfico superiores a las del resto del país, gracias a la oferta laboral de la industria maquiladora. Durante estos años es indiscutible que esta industria ha generado empleos, pero estos han sido con arduas jornadas de trabajo y salarios bajos. Abajo se presenta el número de maquiladoras establecidas en Ciudad Juárez desde el año 2005 al 2015 (ver figura I).

Como consecuencia de la crisis inmobiliaria de Estados Unidos y la incertidumbre generada por la inseguridad, descendió el número de trabajadores empleados por la maquila. La caída del empleo en la industria maquiladora en Ciudad Juárez inició en 2008, y un año después llegó a su punto más bajo con $174 \mathrm{mil}$ 450 empleados (ver figura II).

Ante este complejo escenario, la posibilidad de reactivación planteó un pacto social distinto, más amplio, de ma-
Figura I. Maquiladoras establecidas en Ciudad Juárez en los últimos 10 años.

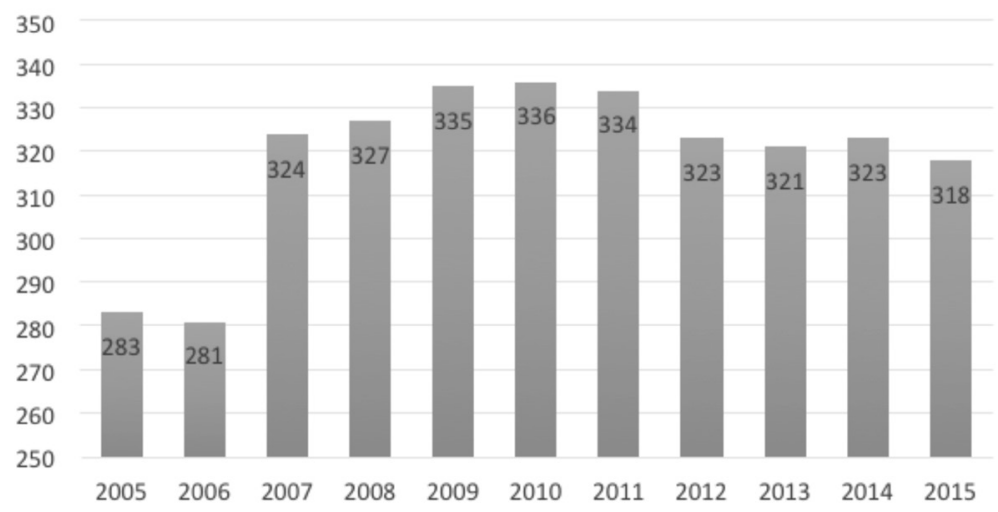

Fuente: Elaboración propia con base en datos proporcionados por INDEX Juárez.

Figura II. Empleos generados por la industria maquiladora en Ciudad Juárez del 2007 al 2015.

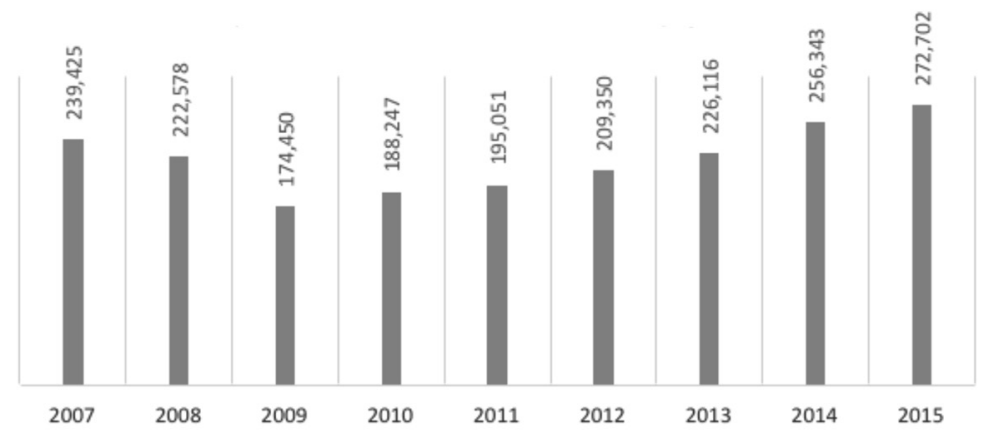

Fuente: Elaboración propia con base en datos proporcionados por INDEX Juárez. 
yor participación de los diferentes niveles de gobierno y de los actores sociales y productivos del estado de Chihuahua y de la región. No es atrevido decir que el surgimiento de un Estado de bienestar "in extenso" se dio con la concurrencia de la federación, del Estado de Chihuahua y del municipio, desplegando políticas públicas sobre temas de seguridad y justicia transversales y una reasignación financiera traducida en objetivos y metas gasto-presupuesto.

Para resolver la situación de inseguridad, que es pluricausal (se fue gestando paulatinamente y tuvo que ver en gran parte con la desintegración y descomposición familiar), la aplicación de la teoría del Estado de bienestar se manifestó a través de una mezcla de acciones de gobierno que atendieron aspectos sociales y jurídicos, entre los que destacan: depuración de cuerpos policiacos, ampliación de infraestructura educativa y espacios para alumnos, programas de intervención comunitaria, acciones contra las adicciones, cambios en la legislación y una gran inversión social llevada de la mano con las organizaciones de la sociedad civil.

Fue de gran ayuda que la sociedad se organizara para sumarse en resolver la problemática, se empezaron a realizar una serie de acciones para reparar el tejido social en la ciudad, tales como la participación ciudadana, rescate de espacios públicos, la atención a la infancia, programas de empleabilidad juvenil, se establecieron nuevos centros comunitarios y se dio una especial atención a los adultos mayores.

De manera paralela, la participación de la sociedad civil, mediante esquemas de compromiso en organización e inversión, la apuesta de agentes del sector productivo y social fue gradualmente avanzando hacia una reconversión que elevó la tasa de productividad y el empleo. Como resultante de las medidas aplicadas y los logros gradualmente obtenidos, la inseguridad fue menguando y la confianza ciudadana recobró espacios perdidos, en una comunicación más abierta con sus autoridades al denunciar y participar de manera conjunta con el gobierno.
Según datos de Index Juárez (2015), gracias a la reducción de los índices delictivos, hoy en día la industria maquiladora en Ciudad Juárez recuperó los poco más de 80 mil empleos perdidos desde 2008, cuando la violencia era el tema principal. La ciudad cuenta actualmente con 327 maquiladoras del ramo automotriz, electrónica, eléctrica y médica, procedentes de Estados Unidos, Asia y Europa. A principios del año 2015 el sector maquilador sumó 272072 trabajadores contra el rango más bajo que tuvo en los últimos años que fue de 174450 en 2009.

Para Ampudia (2009), las maquiladoras crearon la paradoja del crecimiento en Ciudad Juárez; por un lado, generaron empleo de baja calificación y derrama salarial de bajos ingresos en la localidad, al grado de nutrir la formación de cinturones de miseria. Además, desde 1993 se empezó a notar la gravedad del problema revelada en la violencia; primero hacia las mujeres, con los feminicidios, después el problema hacia la violencia intrafamiliar y maltrato infantil, para después en 2008 vivir la desbordada violencia, que 
trajo como consecuencia un enorme riesgo hacia la catástrofe social de nuestra ciudad.

En 2008 el gobierno federal implementó una estrategia para detener el crimen organizado que lo único que trajo consigo fue una ola de muertes en la ciudad. La figura 1 muestra el total de defunciones en Ciudad Juárez según el INEGI (2010), desde 1994 hasta 2013, y aunque esta cifra corresponde al total de defunciones y no solo a los casos de homicidios, que se verán más adelante, se puede notar un aumento considerable de muertes en los años 2008 (7911), 2009 (8557) y 2010 (10 143). A partir de 2011 estas estadísticas empiezan a descender culminando en el 2013 con 7257 defunciones (ver figura III).

Entre 2008 y 2011 se registraron más de 9 mil homicidios en Ciudad Juárez, convirtiéndola en la ciudad más violenta del país. Gracias a diferentes acciones emprendidas, esta situación ha cambiado y para el 2014 solo se documentaron 427 homicidios (ver cuadro 1$)$.

A través del Estado de bienestar se alinearon los agentes

Figura III. Defunciones en Ciudad Juárez desde 1994 hasta 2013.

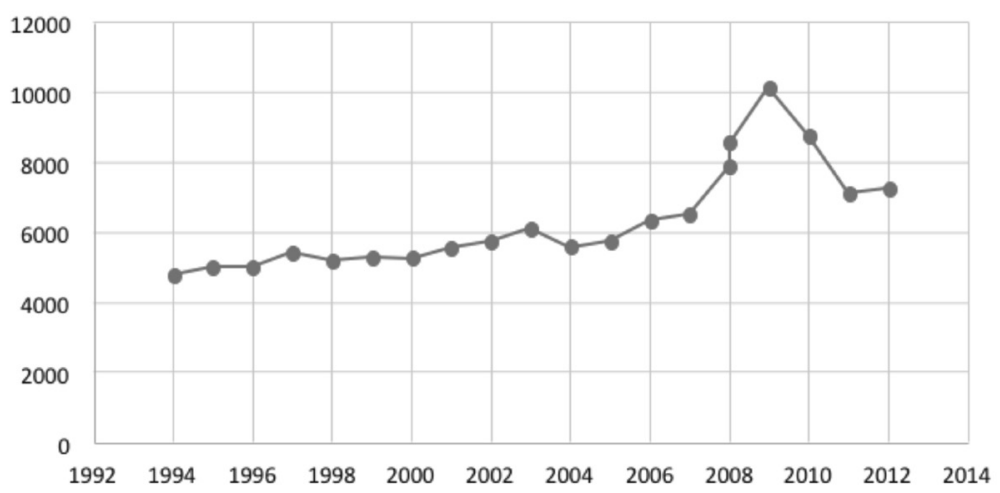

Fuente: Elaboración propia con base a estadísticas del INEGI (2010).

Cuadro 1. Total de homicidios en Ciudad Juárez de 2008 a 2014.

\begin{tabular}{ccccccc}
\hline 2008 & 2009 & 2010 & 2011 & 2012 & 2013 & 2014 \\
\hline 1614 & 2442 & 3101 & 1897 & 503 & 485 & 427 \\
\hline
\end{tabular}

Fuente: Elaboración propia con base en datos del Observatorio de Seguridad y Convivencia Ciudadana del municipio de Ciudad Juárez.

públicos, privados y sociales que dimensionaron las posibilidades de crecimiento de la ciudad en un clima de aceptable seguridad y convivencia, reconociendo deficiencias, pero valorando el esfuerzo compartido. Vale la pena hacer un recorrido histórico sobre la conceptualización del Estado de bienestar para entender su importancia en la recomposición del tejido social en Ciudad Juárez.
EL EMPRENDIMIENTO SOCIAL COMO ALTERNATIVA AL ESTADO DE BIENESTAR

ada vez es más común la tendencia de los países para dejar de ser un estado benefactor alejado de los programas sociales. El emprendimiento social crea empleo y además se asegura de mejorar la calidad de vida de las personas. Este adelgazamiento del estado de bienestar debe suplirse con otras alternativas para no dejar despro- 
tegida a la población. Una de estas alternativas es el emprendimiento social que se genera en muchas ocasiones por el autoempleo colectivo.

Para Melian y Campos (2010), el fracaso o limitado éxito de las políticas tradicionales de empleo, han favorecido la creación de nuevas estrategias dentro del desarrollo local que utilizan como principal instrumento para la creación de empleo estable y de calidad el impulso de Pymes, muchas de las cuales se crean en forma de economía social: Cooperativas de Trabajo Asociado, Sociedades Laborales, Asociaciones y Fundaciones, etcétera. Estas empresas favorecen la inserción sociolaboral de los grupos vulnerables en el mercado de trabajo y producen efectos positivos como mayor cantidad y calidad del empleo y crecimiento y desarrollo equilibrado y sostenible.

Ante las crisis financieras que están surgiendo en los gobiernos y que limitan el estado de bienestar, los emprendedores sociales nacen no solo para mejorar la economía, sino lo social y con esto se crean sociedades más justas.

\section{Conclusiones}

- Los programas sociales han mejorado las condiciones de vida de sus usuarios y servido para poner en marcha procesos de acompañamiento e inserción social. No puede dejarse de lado que la valoración de las personas beneficiarias suele ser, por lo general, positiva y altamente satisfactoria con respecto a dichos programas.

- El Estado de bienestar se fundamenta en la tesis de que los gobiernos tienen la responsabilidad frente a su población de proporcionar una vida digna, creando instituciones y programas sociales para enfrentar las enfermedades, la pérdida del trabajo, la discapacidad, la violencia o los bajos salarios que no les permitan a los ciudadanos un nivel de bienestar.

- Se debe pensar en construir un nuevo Estado de bienestar del siglo XXI, con la experiencia del pasado pero con visión de futuro, en el que las políticas sociales no estén reñidas con la viabilidad financiera del gobier- no, donde los ciudadanos tengan un compromiso y se genere un emprendimiento social que beneficie a la economía de la población.

- Se ha confirmado que el Estado del bienestar genera beneficios no solo para las personas de manera individual, sino para la sociedad en su conjunto. Frente a las necesidades del mundo en desarrollo, parece necesaria la creación de un nuevo modelo de Estado benefactor, capaz de responder a las condiciones de una economía globalizada y a dinámicas de mercado que no siempre visualizan el impacto de sus actividades entre los sectores más desfavorecidos de la sociedad.

- La tipología del Estado de bienestar es adecuada para contrastar la evolución de Ciudad Juárez, como centro de desarrollo fronterizo. Las políticas públicas de beneficio social asignadas a la ciudad permiten revisar su complejidad traducida en su actividad económica, particularmente la industria maquiladora, en donde se observó una disminución del reclutamiento de 
personal, los homicidios asociados a la crisis de violencia e inseguridad que se padeció.

- Tanto en Ciudad Juárez como en otras ciudades fronterizas de México, la teoría del Estado de bienestar y su aplicación práctica, son necesarias para generar crecimiento y desarrollo, así como para la regeneración de la regularidad en la vida social en sus actividades económicas, productivas, sociales, prestación de servicios públicos y atención y gestión de otras medidas comunitarias que se articulan con las organizaciones de la sociedad civil, es aquí donde el emprendimiento social tiene sentido para crear empleo y mejorar la calidad de vida de las personas.

- El Estado del bienestar cobra mayor importancia en los periodos de crisis; en el caso de Ciudad Juárez esta situación se dio tanto en materia de seguridad como económica. Es de esperar que los beneficios de su aplicación se vean reflejados en el mantenimiento de la paz social y en un mejor nivel de vida de sus habitantes.

\section{REFERENCIAS}

\section{BIBLIOGRÁFICAS}

Ampudia, M. (2009). Empleo y estructura económica en el contexto de la crisis de Ciudad Juárez: las amenazas de la pobreza y la violencia. Diagnóstico sobre la realidad social, económica y cultural de los entornos locales para el diseño de intervenciones en materia de prevención y erradicación de la violencia en la región norte: el caso de Ciudad Juárez, Chihuahua. México. Capítulo I. Comisión nacional para prevenir $\mathrm{y}$ erradicar la violencia contra las mujeres.

Bustelo, E. \& Minujin, A. (1997). La política social esquiva. Revista de Ciencias Sociales, 6,7- 55.

Comisión Nacional de Población (2008). Informe de México: El cambio demográfico, el envejecimiento y la migración internacional en México. Comité especial sobre población y desarrollo. XXXII periodo de sesiones de la Comisión Económica para América Latina y el Caribe (CEPAL). http://www.conapo.gob. $\mathrm{mx} / \mathrm{en} / \mathrm{CONAPO} / 25$ de_Junio_de_2008

Comisión Nacional de Población (2012). Índice de Marginación Urbana 2010. México: CONAPO. Recuperado de http:// www.conapo.gob.mx/ es / CONAPO/Capitulo_1_Marginacion_Urbana_2010

Crozier, M., Hutington, S. P., \& Watanuki, J. (1975). The Crisis of Democracy: Report on the Governability of Democracies to the Trilateral Commission. New York: NY University Press. Esping-Andersen, G. (1993). Los tres mundos del Estado de Bienestar. Valencia: Ediciones Alfons el Magnani.

Euzeby, C. (1991). La renta mínima en los países de la comunidad europea. Experiencias y perspectivas. IV Jornadas de Economía de los Servicios Sociales. Gobierno Vasco, VitoriaGasteiz.

Index Juárez (2015). Asociación de Maquiladoras A.C. http://indexjuarez.org/

Instituto Municipal de Investigación y Planeación. (2003). Plan de desarrollo 
urbano de Ciudad Juárez. Ciudad Juárez, Chihuahua. Instituto Nacional de Estadística y Geografía. (2010). Cuéntame. Recuperado de: http://cuentame.inegi. org. $\mathrm{mx} /$ poblacion/esperanza.aspx?tema $=\mathrm{P}$

Instituto Nacional de Estadística y Geografía. (2013) Recuperado de: http:// www3.inegi.org.mx/sistemas/mexicocifras/default. aspx ? $=08$

Krmpotic, C. (2002). La Conferencia Nacional de Asistencia Social de 1933. Los debates en torno al progreso, la pobreza y la intervención estatal. Scripta Ethnologica, 24, 37-57.

Lodemel, I. \& Trickey, H. (2001). An offer you can't refuse. Workfare in international perspective, Policy Press, Bristol.

Luhmann, N. (1993). Teoría Política del Estado de Bienestar (p. 32). Madrid: Alianza Editorial.

Martínez, Jorge (2000). El Destino de las Naciones. Barcelona: Ariel.

Melián A. y Campos V. (2010) Emprendedurismo y economía social como mecanismos de inserción sociolaboral en tiempos de crisis. REVESCO. Revista de Estudios Cooperativos (100). pp. 43-67. ISSN 1885-8031

Moreno, G. (2008). La reformulación del Estado del bienestar: el workfare, las políticas activas de empleo y las rentas mínimas. Ekainia, 43, 143-154.

Moreno, L. (2001). Estado del Bienestar y mallas de seguridad. Madrid: CSIC.

Mulas-Granados, C. (2010). El Estado dinamizador. Nuevos riesgos, nuevas políticas y la reforma del Estado de bienestar en Europa. Madrid: Universidad Complutense de Madrid.

Observatorio de Seguridad y Convivencia Ciudadanas del Municipio de Ciudad Juárez, Chihuahua. (2015). Observatoriodejuarez.org

Pico, J. (1999). Teorías sobre el Estado de bienestar. Madrid: Siglo XXI.

Sen, A. (2002). ¿Por qué la equidad en salud? Revista Panamericana de la salud pública, 11 (5-6), 302-309.

Villarespe, V. (2002). Pobreza: Teoría e historia. Instituto de Investigaciones Económicas. México: UNAM. 